\title{
Bremsstrahlung Background Modeling Without Fit Regions
}

Frank Eggert $^{1}$ and Patrick Camus ${ }^{2}$

${ }^{1}$ AMETEK / EDAX, Mahwah, New Jersey, United States, ${ }^{2}$ AMETEK, Mahwah, New Jersey, United States

The Bremsstrahlung background is a major part of all EDS spectra. It is a fundamental requirement to consider this with any EDS spectra evaluation. Two very different strategies have prevailed. One pure mathematical way is to apply filtering of the spectrum to suppress all background parts [1]. Another more physical theory-based method is to calculate the bremsstrahlung background [2,3] based on the almost 100 year-old fundaments by Kramers [4]. This method is also the only choice for complete spectra simulation based on model equations (to distinguish from full Monte Carlo based simulation of spectra which includes also continuous X-ray simulation).

The physical equations-based calculation of the bremsstrahlung background has already a very long history. A well-known approach for the background calculation was introduced by Lifshin [2] who has extended the physics equation with a doubled Kramers equation and with a requirement to fit two open parameters at two selected points with measured spectrum. It was well-known and it was also shown by Statham [5] that this way with a two-points adjustment of the bremsstrahlung equations is not matching real spectra in all circumstances. A modified approach provides better results with using only one or as many fit regions as desired [6,7]. This is coupled with a search for possible fit regions where no characteristic lines are located. But sometimes it might be a disadvantage to require background regions where the model needs to be fitted to the spectrum, despite the ability of modern algorithms to determine the required fit regions automatically.

Heckel/Jugelt [8] investigated the absolute determination of the bremsstrahlung spectrum with the aim that only one absolute adjustment with the measured spectrum is required to reproduce the entire bremsstrahlung distribution. This way was used for spectra evaluation application by the same authors [9] and also by Trincavelli et al. [10] Simulations of EDS spectra need improved bremsstrahlung models to calculate bremsstrahlung distribution with only one absolute adjustment to the measured spectra $[11,12]$. Because the complete modelling of a bremsstrahlung distribution of EDS spectra is possible for simulation, it is then also possible to use for spectra evaluation with no further need for mathematical bending of the physical model-based calculation by background regions fitting. An overall absolute adaption to the measured spectrum is still required. But this is then performed without user interaction and it is no longer necessary for the user to provide any fit regions. The frequently mentioned disadvantage with the bremsstrahlung model-based background calculation vs. filtering has disappeared. The benefits are with the modelling to investigate and to consider sample surface roughness, sample tilts and with TEM also of the sample thickness [7]. Filtering loses this analytically important information. In cases of bad background fits, the method for absorption path optimization is possible to use. The bremsstrahlung calculation is then optimized by a physical model parameter change, the absorption in sample. 


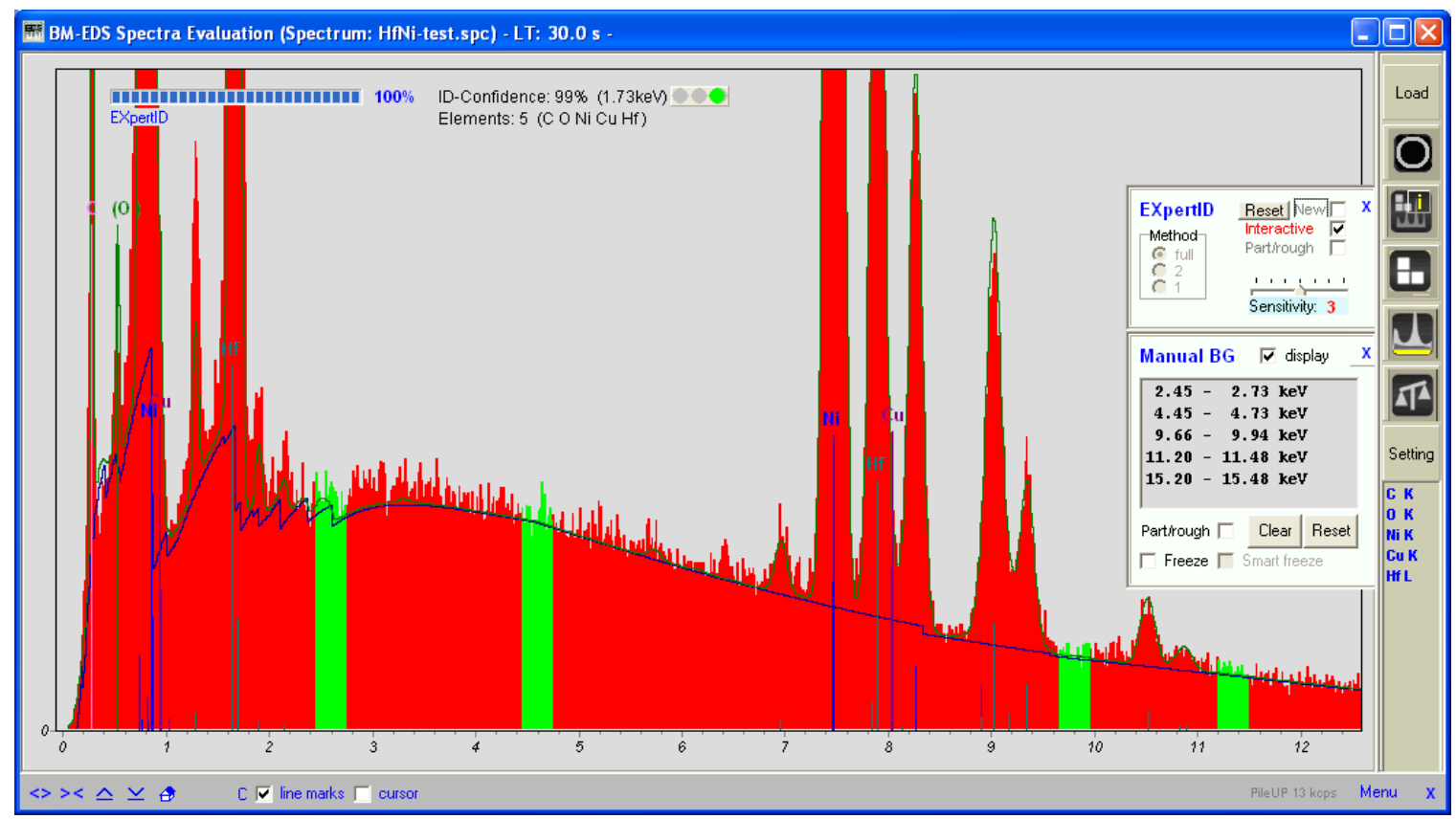

Figure 1. Background approximation with automatically determined fit regions (based on a peak search to find regions without characteristic lines).

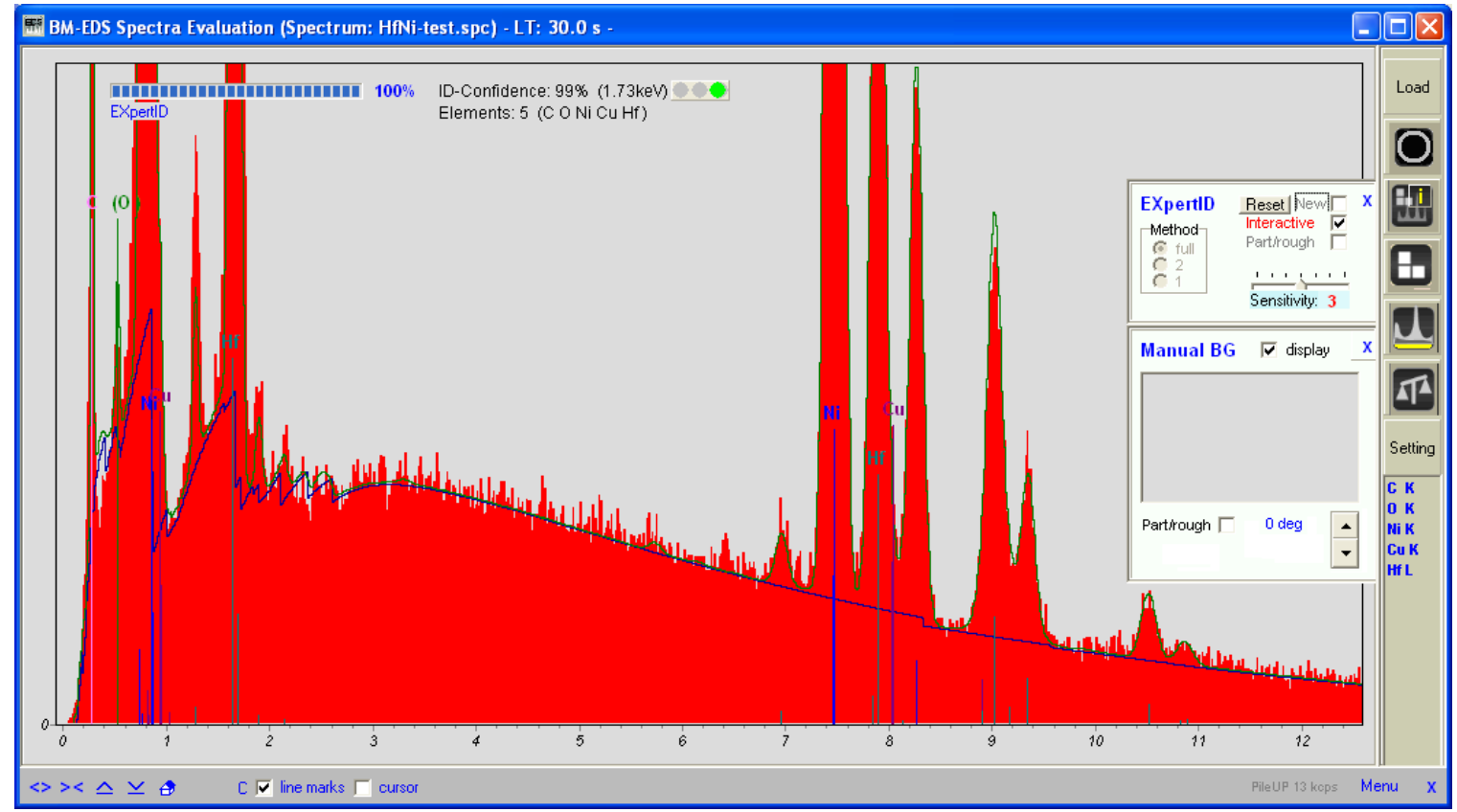

Figure 2. Same spectrum but with an overall absolute adjustment without BG points fitting.

\section{References}

[1] F H Schamber, Proc. $13^{\text {th }}$ Nat. Conf. Microbeam Analysis Soc. (1978) 50

[2] E Lifshin, Proc. $9^{\text {th }}$ Nat. Conf. Microbeam Analysis Soc. (1974) 53

[3] C E Fiori, R L Myklebust, K F J Heinrich, H Yakowitz, Anal. Chem. 48 (1976) 172

[4] H A Kramers, Philos. Mag. 46 (1923) 836

[5] P J Statham, J. Res. Natl. Inst. Stand. Technol. 107 (2002) 531 
[6] F Eggert F Experim. Techn. d. Physik 33 (1985) 441

[7] F Eggert, P P Camus, M Schleifer, F Reinauer, IOP Conference Series: Materials Science and Engineering 304 (2018)

[8] J Heckel, P Jugelt, Experim. Techn. d. Physik 31, 6 (1983) 493

[9] J Heckel, P Jugelt, X-Ray Spectrom. 16 (1987) 67

[10] J Trincavelli, G Castellano, J A Riveros, X-Ray Spectrom. 27 (1998) 81

[11] P Duncumb, P J Statham, Microchim. Acta 138 (2002) 249

[12] F Eggert Microchim. Acta 155 (2006) 129 